\title{
CARACTERÍSTICAS DE LA COMPETENCIA PROSÓDICA DE JÓVENES BILINGÜES VASCOS EN LA LECTURA EN VOZ ALTA: LAS CUMBRES TONALES
}

\author{
YOUNG BASQUE BILINGUALS' PROSODIC COMPETENCE \\ FEATURES IN READING ALOUD: THE TONAL PEAKS
}

\author{
IÑAKI GAMINDE \\ Universidad del País Vasco/EHU \\ inaki.gaminde@ehu.es \\ AINTZANE ETXEBARRIA \\ Universidad del País Vasco/EHU \\ aintzane.etxebarria@ehu.es \\ ASIER ROMERO \\ Universidad del País Vasco/EHU \\ a.romero@ehu.es

\section{NAIA EGUSKIZA} \\ Universidad del País Vasco/EHU \\ eguskiza.n@gmail.com
}

\section{RESUMEN}

El objetivo de este trabajo es describir las relaciones entre las distintas cumbres tonales de un grupo prosódico en la lengua vasca, para la identificación de diferentes modelos combinatorios de cumbres y la relación y el uso de los modelos según factores endógenos (puramente lingüísticos) y exógenos, como el género, el grupo lingüístico y la procedencia de los informantes. Para la confección del corpus se ha utilizado un texto leído por 101 jóvenes bilingües vascos de ambos géneros, con lengua materna euskera y español, en unos casos, y euskera y francés, en otros. Algunos de ellos han aprendido el euskera en el entorno familiar y otros en la escuela, todos ellos son procedentes de los territorios del País Vasco. El análisis de los datos se ha realizado en tres niveles: en el nivel léxico, sintáctico y

* Este trabajo ha sido financiado en el marco de dos proyectos de investigación: Gobierno Vasco [IT 1028/16] y Universidad del País Vasco [GIU 22/16]. 
en el del grupo prosódico. Una vez realizado el análisis se concluye que no hay diferencias estadísticamente significativas entre los tres niveles citados; las interacciones de todos los niveles muestran variedad y dispersión en cuanto a la cumbre tonal; y, por último, la manera en la que los informantes han aprendido la lengua vasca y la procedencia geográfica influye en la posición de la cumbre tonal.

Palabras clave: Lengua vasca, competencia prosódica, cumbres tonales, lectura en voz alta.

\section{ABSTRACT}

The purpose of this paper is to describe the relation between the different tonal peaks of a prosodic group in Basque language to identify the different combination models of peaks and the relation and use of the models according to endogenous (linguistics) and exogenous (gender, linguistic group, provenance) factors. To create this corpus a text read by 101 bilingual young men and women has been used. Their mother tongue was Basque and Spanish in some cases and Basque and French in other cases. They come from the Basque Country territories, some learnt Basque within the family environment and others at school. Data analysis has been carried out in three levels: on the lexical level, at syntactic level and in the prosodic group. The conclusions are that there are no statistically significant differences according to the three levels mentioned; the interaction of all the levels show variety and dispersion referring the tonal peak and, finally, the position of the tonal peak is influenced by the way the informants have learnt Basque as well as their geographical origin.

Keywords: Basque language, prosodic competence, tonal peaks, reading aloud.

Recibido: 03.11.2016. Aceptado: 17.05.2017.

\section{INTRODUCCIÓN}

厂 a prosodia es un conjunto de rasgos suprasegmentales en los que se incluyen el acento, la entonación, el tono, el ritmo, la melodía, las pausas, la velocidad de elocución y la cualidad de la voz (Llisterri, 2016). Un hablante debe desarrollar una adecuada competencia prosódica para dar información sobre el estado emocional en general y su actitud con respecto a un tema determinado, a una situación en concreto o acerca del interlocutor (Mozziconacci, 1998). Además, aporta datos de tipo lingüístico sobre la estructura oracional por medio de la distribución tonal y la tipología pausal, e informa sobre el estatus, la distancia social y las relaciones de poder entre los interlocutores (Fujisaki, 2004). Se debe subrayar que un uso adecuado de la prosodia no solamente atañe al habla espontánea, sino que tam- 
bién a una correcta lectura en voz alta, puesto que influye en la comprensión tanto de lo que se lee como de lo que se escucha, tal y como se describe en numerosos estudios:

Conveying meaning by the use of prosody is not only important in speech. When reading text out loud, a correct use of prosody by the reader makes text sound more natural and comprehensible to the listener (e.g., Kuhn y Stahl, 2003; Rasinski, 2004). Prosody is the most recent addition to the components of text reading fluency, together with reading speed and reading accuracy (e.g., Dowhower, 1991; Kuhn y Stahl, 2003). There is a growing literature on the relationship between reading fluency and reading comprehension that emphasizes the role of text reading prosody (e.g., Klauda y Guthrie, 2008; Miller y Schwanenflugel, 2006, 2008; Rasinski, Rikli y Johnston, 2009) (Veenendaal, Groen, y Verhoeven, 2014: 521).

La entonación como parte de la prosodia "es la sensación perceptiva que produce, fundamentalmente, las variaciones de tono a lo largo de un enunciado" (Gil, 2007). Este rasgo señala la modalidad oracional, la información nueva en el discurso y la función pragmática y, además, enfatiza los elementos del discurso y aporta información sobre aspectos de cortesía (Llisterri, 2016). Prieto (2002) atribuye tres tipos de funciones a la entonación, que son la función expresiva, ya que es un recurso modalizador; la función focalizadora, dado que el hablante selecciona la información central que desea enfatizar; y la función demarcativa, ya que el emisor divide el texto en unidades tonales.

En la literatura científica sobre la prosodia en la lectura en voz alta se estudia la importancia de la entonación como rasgo de expresividad que aporta el nivel más alto de comprensión. En esta línea, se señala que la variación entonativa aporta información sobre el significado de la oración, para ello la distribución de las pausas tiene que coincidir con la estructura sintáctica y el contorno tonal al final de la oración debe ser el adecuado (Benjamin y Schwanenflugel, 2010; Miller y Schwanenflugel, 2006, 2008; Benjamin, Schwanenflugel, Meisinger, Groff, Kuhn y Steiner, 2013). En definitiva, elementos suprasegmentales como la entonación y el acento se han considerado esenciales para una lectura con fluidez, tal y como señalan Miller y Schwanenflugel (2008) apoyados en las investigaciones de algunos autores (Chomsky, 1978; Rasinski, 1990; Samuels, Schermer y Reinking, 1992). Además, ambos componentes contribuyen a reflejar el nivel de comprensión que se muestra a través de una correcta agrupación sintáctica de los elementos de un texto (Kuhn y Stahl, 2003; Miller y Schwanenflugel, 2008). En algunos trabajos que han comparado a lectores con fluidez, con aquellos lectores que no tienen esta característica, se ha visto que varía el tipo de tono empleado en las oraciones declarativas y algunas interrogativas: 
Fluent readers read with larger downward pitch changes for declarative sentences and larger pitch rises for yes-no questions than do less fluent readers (Miller y Schwanenflugel, 2006; Schwanenflugel, Hamilton, Wisenbaker, Kuhn y Stahl, 2004; Benjamin et al., 2013: 106).

Respecto a la lengua vasca, por ahora no se han llevado a cabo trabajos que permitan distinguir entre lectores con fluidez y sin fluidez. Asimismo, tampoco hay suficientes estudios que aporten datos sobre la influencia de los rasgos suprasegmentales en la comprensión textual, a pesar de que han sido numerosas las investigaciones sobre la entonación de la lengua vasca que han descrito el elemento focalizado de las oraciones en diversas variedades de la lengua vasca (Elordieta, 2007a, 2007b; Ito, 2002, 2003) y en el modelo estándar (Gaminde, 2010). Además, siguiendo el modelo métrico autosegmental de notación prosódica ${ }^{1}$ (Pierrehumbert, 1980; Beckman y Pierrehumbert, 1986; Pierrehumbert y Beckman, 1988; Liberman y Sag, 1975; Prieto, 2002) y teniendo en cuenta la posición del acento se han especificado combinaciones tonales de oraciones enunciativas, interrogativas y negativas para la lengua estándar, por ejemplo siendo para la oración "laguna sartu da" (ha entrado el amigo) la siguiente: \% $\%\left[\mathrm{~L}^{*}+\mathrm{H}_{-} \mathrm{H}^{*}+\mathrm{L}-\mathrm{L}+\mathrm{H}^{*}\right]$, $\mathrm{L} \%$ para las oraciones enunciativas y $\% \mathrm{~L}\left[\mathrm{~L}^{*}+\mathrm{H}-\mathrm{L}^{*}+\mathrm{H}-\mathrm{L}+\mathrm{H}^{*}\right] \mathrm{HL} \%$ para las interrogativas de tipo Sí/No o absolutas (Gaminde, Aurrekoetxea, Etxebarria, Garay y Romero, 2014). Cabe destacar que para el estudio de la entonación de la lengua vasca a menudo se han empleado oraciones aisladas o textos leídos (Hualde, Elordieta y Elordieta, 1994; Uriarte, 1995, 2004; Gaminde, 1995; Gandarias, 1996; Elordieta, 1997, 1999; Jun y Elordieta, 1997; Gaminde, 2000, 2003; Hualde, 1999; Navas, 2003; Gaminde et al., 2014), pero se ha trabajo muy poco con textos espontáneos (Gaminde, 2004a, 2004b).

Dadas las dificultades que en general se han detectado en euskera para conocer la posición de la sílaba acentuada debido a las diferentes variedades diatópicas, actualmente se ha optado por un modelo mixto de análisis, que aunque utiliza la notación del modelo métrico autosegmental, toma como base el análisis fonético de la curva de f0 dentro del grupo prosódico (Gaminde, Etxebarria, Romero y Eguskiza, 2016), y que se define como una unidad entonativa comprendida entre dos pausas (Gaminde, 2004a; Gaminde, 2007; Gaminde, Etxebarria, Garay y Romero, 2012; Gaminde et al., 2014), su extensión, teniendo en cuenta el número de sílabas, varía según se trate de un texto leído o espontáneo, puesto que según se ha demostrado en varias investigaciones (Gaminde, 2004a, 2006, 2007, 2010; Gaminde y Goikoetxea, 2005; Gaminde et al., 2012; Gaminde, Etxebarria, Garay

${ }^{1}$ Hualde define el modelo métrico autosegmental de notación prosódica de la siguiente manera: "La identificación de los elementos contrastivos del sistema entonativo cuya combinación produce los contornos melódicos que encontramos en los enunciados posibles de la lengua” (Hualde: 2003: 1). 
y Romero, 2013) el porcentaje de los grupos prosódicos cortos es mayor en textos espontáneos que en los leídos.

La entonación de los grupos prosódicos, independientemente de su longitud, se percibe como una sucesión de cambios en la altura tonal (agudo/grave) del enunciado llamado melodía (Llisterri, 2016), estos picos o cumbres tonales, siguiendo la teoría de la jerarquía prosódica ${ }^{2}$, se dan en distintas sílabas y en diferentes niveles: léxico, sintáctico y grupo prosódico. De todas formas, aún están por estudiar las características melódicas de estas unidades en los textos tanto leídos como espontáneos. Por lo tanto, el objetivo de este trabajo es la descripción de las relaciones entre las diferentes cumbres prosódicas de un grupo prosódico, para la identificación de diferentes modelos combinatorios de cumbres y la relación y el uso de los modelos según factores endógenos (puramente lingüísticos) y exógenos, como el género, el grupo lingüístico (A el euskera se ha aprendido en el entorno familiar y B en la escuela) y la procedencia de los informantes.

\section{MÉTODO}

Para reunir el corpus objeto de estudio tomaron parte 101 jóvenes bilingües vascos, de éstos 10 hablan el vasco y el francés, y 91 el vasco y el español. En cuanto al género, 46 son de género masculino y 55 del femenino y todos ellos tienen una edad comprendida entre los 18 y 25 años. Respecto a la lengua vasca, algunos de ellos la han aprendido en el entorno familiar (56) y otros en la escuela (45), ésta es la razón por la que se distinguen los grupos A y B.

Para la confección del corpus todos los informantes leyeron la siguiente noticia deportiva en voz alta, después de una primera lectura en voz baja:

"Urte asko pasatu beharko dira beste pilotari batek Aimar Olaizolari pilotari onenaren titulua kentzeko. Zazpi final jokatu ditu, zazpi txapel jantzi eta kaiolan etorkizuneko belaunaldiek bideoan miretsiko duten abilezia lortu. Hori ez du lortu bakarrik jokatuz, aurkari gogorrei aurre eginez baizik. Horixe falta zitzaion txapeldun handiari, gogoan hartzeko moduko final bat" (Berria, 2013) ${ }^{3}$.

Las grabaciones se realizaron con las grabadoras Marantz PMD620 y ZOOMH4, y con un micrófono externo.

El grupo prosódico elegido para su análisis fue el siguiente, puesto que en las

2 "As is well-known, there are distinct stress patterns associated with a) lexical stress, b) compound stress, and c) phrasal stress" (Nespor \& Vogel, 2007).

${ }^{3}$ La traducción del texto leído es la siguiente:

"Pasarán muchos años para que otro pelotari le pueda quitar el premio de mejor pelotari a Aimar Olaizola. Se han jugado siete finales, se han dado siete premios, y se ha logrado una habilidad que las futuras generaciones la admirarán por medio del vídeo. Todo esto no lo ha conseguido jugando solo, sino que ha tenido que competir contra duros adversarios. Esto es precisamente lo que necesitaba el gran campeón, una final para recordar”. 
realizaciones de una gran parte de los informantes, coincidía tanto en el número de sílabas como en el número de elementos del grupo prosódico:

"Horixe falta zitzaion txapeldun handiari".

Este grupo prosódico fue realizado como un único enunciado por 75 de los 101 informantes, y consta de tres sintagmas: uno (Horixe) compuesto de un pronombre intensivo, que es el foco de la frase, y dos sintagmas compuestos. No se han utilizado las producciones del resto de los informantes porque realizaron algún tipo de pausa que rompía la estructura del grupo prosódico:

$\begin{array}{lll}\begin{array}{l}\text { falta } \\ \text { faltar (par) } \\ \text { "le faltaba" }\end{array} & \text { zitzaion } \\ & \text { AUX bivalente } & \\ \text { txapeldun } & \text { handi } & \text {-ari } \\ \text { campeón (Nom.) } & \text { gran (Adj.) } & \text { dat. sing. } \\ \text { al gran campeón } & & \end{array}$

El corpus se etiquetó y se transcribió con el programa Praat (Boersma y Weenink, 2009). Para realizar el análisis de los datos, las señales se etiquetaron en tres niveles. En el nivel léxico se estudió en qué sílaba se encuentra la cumbre prosódica de la palabra; en el nivel sintáctico se analizó en qué constituyente de los dos sintagmas compuestos se realiza la cumbre prosódica dominante de todo el sintagma. Finalmente, en el nivel del grupo prosódico (GP) se consideró en qué sintagma se realiza la cumbre prosódica que domina todo el grupo.

Las variables que se tienen en cuenta en el análisis de los datos son el género, la lengua materna y la procedencia geográfica que se presenta dividida en tres zonas: la occidental, que comprende las provincias de Araba y Bizkaia; la central, que engloba las provincias de Gipuzkoa y Navarra; y la oriental, comprendiendo la zona del País Vasco continental.

\section{ANÁLISIS DE LOS DATOS}

El análisis de los datos, que se presenta en este apartado, tal y como se ha mencionado en el apartado anterior, se realizó en tres niveles (Figura 1). 


\begin{tabular}{|l|c|c|c|c|c|}
\hline GP & \multicolumn{4}{|c|}{$*$} \\
\hline & \multicolumn{4}{|c|}{ Horixe falta zitzaion txapeldun handiari } \\
\hline Sintaxis & $*$ & \multicolumn{2}{|c|}{$*$} & \multicolumn{2}{c|}{$*$} \\
\hline & horixe & \multicolumn{2}{|c|}{ falta zitzaion } & $*$ & $*$ \\
\hline Léxico & $*$ & $*$ & $*$ & $*$ & $*$ \\
\hline & horixe & falta & zitzaion & txapeldun handiari & handiari \\
\hline
\end{tabular}

Figura 1. Niveles de análisis de las cumbres prosódicas; el símbolo “*” indica la sílaba de la cumbre prosódica en cada nivel.

En el nivel léxico, la cumbre prosódica de “horixe”, primer elemento del grupo prosódico, puede aparecer en la segunda sílaba (tres casos; 4\%) o en la tercera (72 casos; 96\%). En lo que respecta a la cumbre prosódica de "falta", ésta se puede encontrar en la primera sílaba (18 casos; $24 \%$ ) o en la segunda (57 casos; $76 \%$ ). Hay que hacer notar que en este caso las diferencias entre las dos sílabas son muy pequeñas, esto puede ser debido a la posición que ocupa la palabra dentro del grupo prosódico (Figura 2).

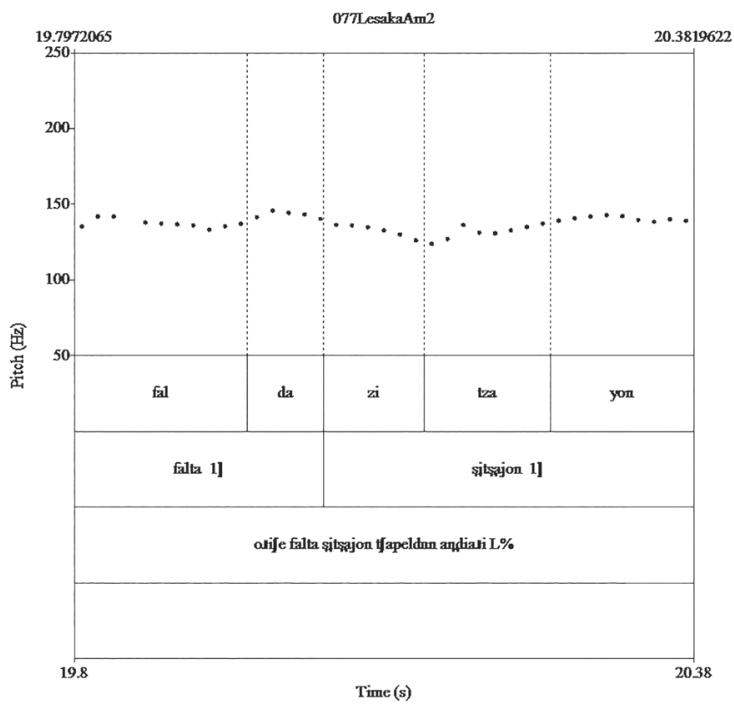

Figura 2. Cumbre prosódica de "falta", la diferencia en f0 entre una y otra sílaba es de $8 \mathrm{~Hz}$ (informante masculino de Lesaka con lengua materna euskera).

La cumbre de "zitzaion” se sitúa principalmente en la tercera sílaba (74 casos; 
98,67\%); sólo se ha constatado un caso donde aparece en la primera sílaba (1, $3 \%)$. En el caso de "txapeldun", la cumbre puede aparecer en la segunda sílaba (tres casos; 4\%) o en la última (72 casos; 96\%). Finalmente, la cumbre de "handiari" puede estar en la primera sílaba (cuatro casos; 5,33\%) o en la segunda (71 casos; 94,67\%). Como se constata en este análisis, se puede decir que no existe una gran variación en las posiciones de las sílabas que tienen las cumbres prosódicas. En la Figura 3 se muestran las principales opciones, que constituyen el $92,27 \%$ de todos los casos.

\begin{tabular}{|l|c|c|c|c|c|}
\hline Cumbres & $*$ & $*$ & $*$ & $*$ & $*$ \\
\hline & $\mid$ & $\mid$ & $\mid$ & $\mid$ & $\mid$ \\
\hline Palabra & horixe & falta & zitzaion & txapeldun & handiari \\
\hline
\end{tabular}

Figura 3. Posiciones de las sílabas que contienen las cumbres prosódicas en el nivel léxico.

De la misma manera, teniendo en cuenta el nivel léxico, la media de los valores en $\mathrm{Hz}$ de las sílabas, la curva estándar que se podría dibujar del grupo prosódico "horixe falta zitzaion txapeldun handiari" es la que se muestra en la Figura 4, los valores de los nodos de esta curva son el valor medio de los $\mathrm{Hz}$ en la sílaba:

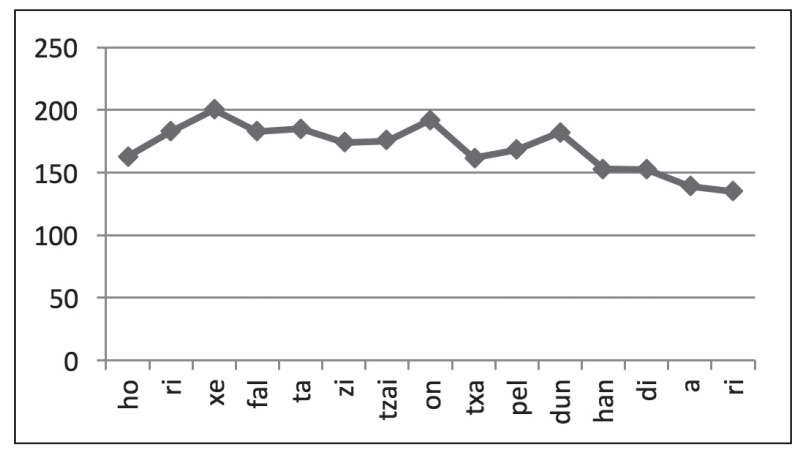

Figura 4. Curva estándar según los valores en $\mathrm{Hz}$ de cada sílaba.

En el nivel sintáctico, se analizó en qué constituyente se sitúa la cumbre prosódica que domina a todo el sintagma. En el caso del sintagma "falta zitzaion", la cumbre principal puede situarse en el primer constituyente (17 casos; 22,67\%) o en segundo (58 casos; 77,33\%). En la Figura 5 se ve la curva con la cumbre pro- 
sódica en el primer constituyente y en la Figura 6 con la cumbre prosódica en el segundo constituyente.

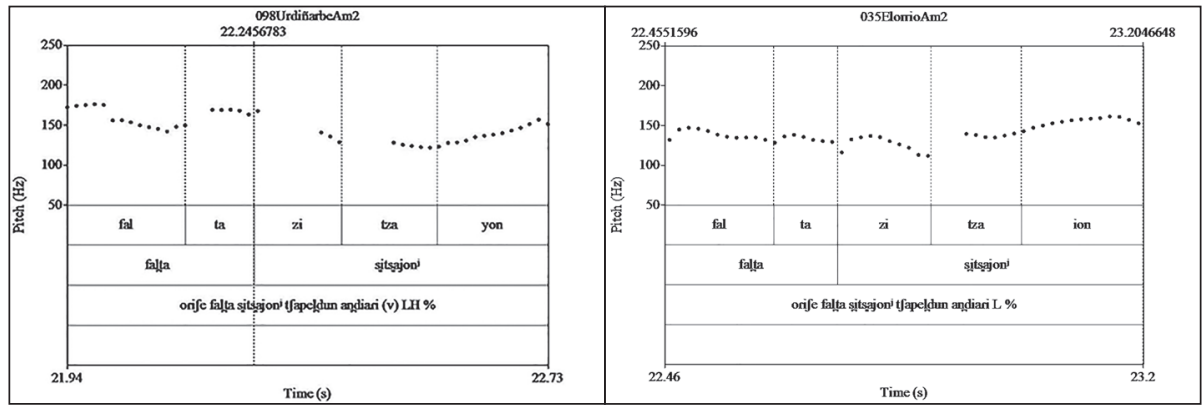

Figura 5. Cumbre prosódica del sintagma "falta zitzaion" en el primer constituyente (informante masculino de Urdiñarbe con lengua materna euskera).
Figura 6. Cumbre prosódica del sintagma "falta zitzaion" en el segundo constituyente (informante masculino de Elorrio con lengua materna euskera).

En lo que respecta al sintagma "txapeldun handiari" la cumbre prosódica sólo aparece en el primer constituyente (Figura 7).

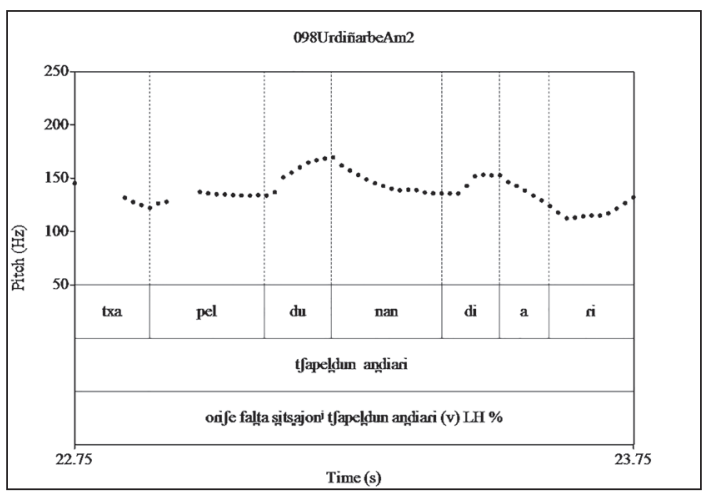

Figura 7. Cumbre prosódica del sintagma “txapeldun handiari” en el primer constituyente (informante masculino de Urdiñarbe con lengua materna euskera).

Como puede comprobarse en el nivel sintáctico tampoco existe una gran variación; en la Figura 8 se muestran los casos más comunes que constituyen el 88,67\% del total. 


\begin{tabular}{|l|c|c|c|c|}
\hline Sintagma & \multicolumn{2}{|c|}{ falta zitzaion } & \multicolumn{2}{c|}{ txapeldun handiari } \\
\hline Cumbres & & $*$ & $*$ & \\
\hline Constituyentes & falta & zitzaion & txapeldun & handiari \\
\hline
\end{tabular}

Figura 8. Constituyentes en los que se realizan las cumbres prosódicas que dominan al sintagma.

Finalmente, la cumbre principal de todo el grupo prosódico puede estar en el primer sintagma ( 59 casos; 78,67\%) o en el segundo (16 casos; $21,33 \%$ ). En la Figura 9 se muestra la curva con la cumbre en el primer sintagma del grupo prosódico; en la Figura 10 la cumbre del grupo prosódico se sitúa en el segundo sintagma.

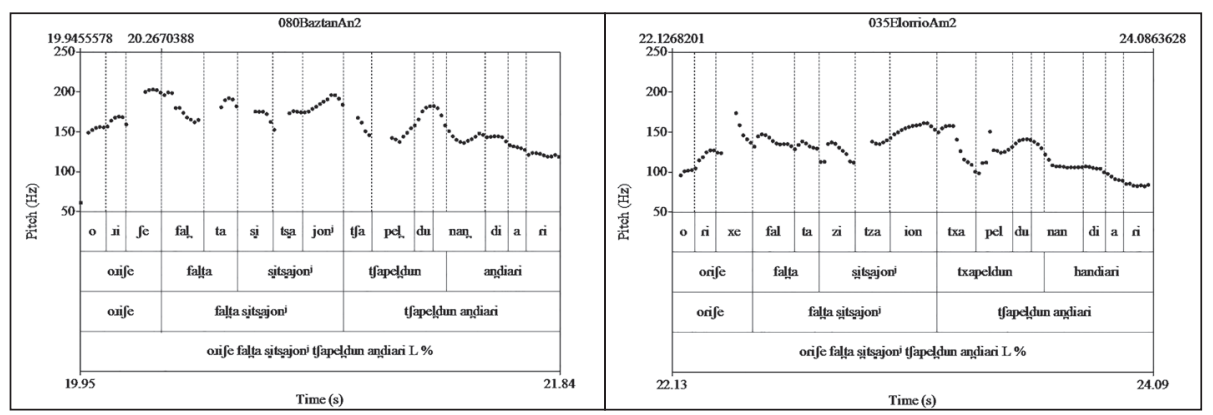

Figura 9. Cumbre del grupo prosódico en el primer sintagma (informante femenina de Baztan con lengua materna euskera).
Figura 10. Cumbre del grupo prosódico en el segundo sintagma (informante masculino de Elorrio con lengua materna euskera).

En los análisis realizados por niveles no se aprecian en un primer momento grandes diferencias, el problema surge cuando se analizaron las interacciones de todos los niveles, es entonces cuando emerge una mayor variedad y los datos aparecen con mayor dispersión. Para el análisis de estas interacciones se realizó una matriz dicotómica, en esta matriz cada variable de las características analizadas anteriormente es un campo. Una vez introducidos los resultados de cada informante, cada uno de ellos aparece constituido por un vector, en estos vectores se muestran las combinaciones posibles que realiza cada informante; estos vectores pueden ser utilizados posteriormente para clasificar a los informantes según las similitudes y disimilitudes que presenten entre ellos.

En la Tabla I se muestran los vectores que emergen del análisis de las interac- 
ciones de los tres niveles. Para la confección de la matriz se han utilizado las ocho características analizadas anteriormente. En las cabeceras de los campos se utilizan las palabras del análisis del nivel léxico, los números indican la posición de la sílaba que porta la cumbre prosódica. Las etiquetas " $1 \sin$ " y " $2 \sin$ " hacen referencia a los sintagmas "falta zitzaion" y "txapeldun handiari", respectivamente; los números indican la posición del constituyente que porta la cumbre prosódica del sintagma. Por último, la etiqueta "CGP" hace referencia a la cumbre del grupo prosódico y los números denotan la posición del sintagma dentro del grupo.

Tabla I. Vectores resultantes del análisis de las interacciones de los tres niveles.

\begin{tabular}{|l|r|r|r|r|r|r|r|r|}
\hline Vector & horixe & falta & \multicolumn{1}{l}{ zitzaion } & txapeldun & handiari & 1Sin & 2Sin & CGP \\
\hline 1 & 3 & 2 & 3 & 3 & 2 & 1 & 1 & 1 \\
\hline 2 & 3 & 2 & 3 & 3 & 2 & 2 & 1 & 1 \\
\hline 3 & 3 & 2 & 3 & 3 & 2 & 2 & 1 & 2 \\
\hline 4 & 2 & 1 & 3 & 3 & 2 & 2 & 1 & 2 \\
\hline 5 & 2 & 2 & 3 & 3 & 2 & 1 & 1 & 1 \\
\hline 6 & 2 & 2 & 3 & 3 & 2 & 2 & 1 & 1 \\
\hline 7 & 3 & 1 & 3 & 3 & 1 & 2 & 1 & 2 \\
\hline 8 & 3 & 1 & 3 & 3 & 1 & 2 & 1 & 1 \\
\hline 9 & 3 & 1 & 3 & 3 & 2 & 1 & 1 & 1 \\
\hline 10 & 3 & 1 & 3 & 3 & 2 & 2 & 1 & 1 \\
\hline 11 & 3 & 1 & 3 & 3 & 2 & 2 & 1 & 2 \\
\hline 12 & 3 & 2 & 1 & 2 & 2 & 1 & 1 & 1 \\
\hline 13 & 3 & 2 & 3 & 2 & 2 & 1 & 1 & 2 \\
\hline 14 & 3 & 2 & 3 & 2 & 2 & 1 & 1 & 1 \\
\hline 15 & 3 & 2 & 3 & 3 & 1 & 2 & 1 & 1 \\
\hline
\end{tabular}

Los quince vectores de la Tabla I tienen unas frecuencias muy desiguales. El vector 2 es el que mayor frecuencia presenta $(32 ; 42,67 \%)$, le siguen los vectores 1 y $3(9 ; 12 \%$ cada uno), a continuación se sitúa el vector 10 (7; 9,33\%), los vectores 9 y 11 ( $4 ; 5,33 \%$ cada uno) y el 15 (2; 2,67\%); los vectores $4,5,6,7,8,12$, 13 y 14 sólo aparecen una vez.

Para realizar la clasificación de los informantes y poder analizar si el género, la lengua materna y la procedencia geográfica de los mismos son relevantes en cuanto al uso de las diferentes características, se realizó un análisis de conglomerados jerárquicos. Para el análisis se ha utilizado la distancia euclídea al cuadrado y el 
método de conglomeración de Ward. En el dendograma de la Figura 11 se muestra la clasificación obtenida.

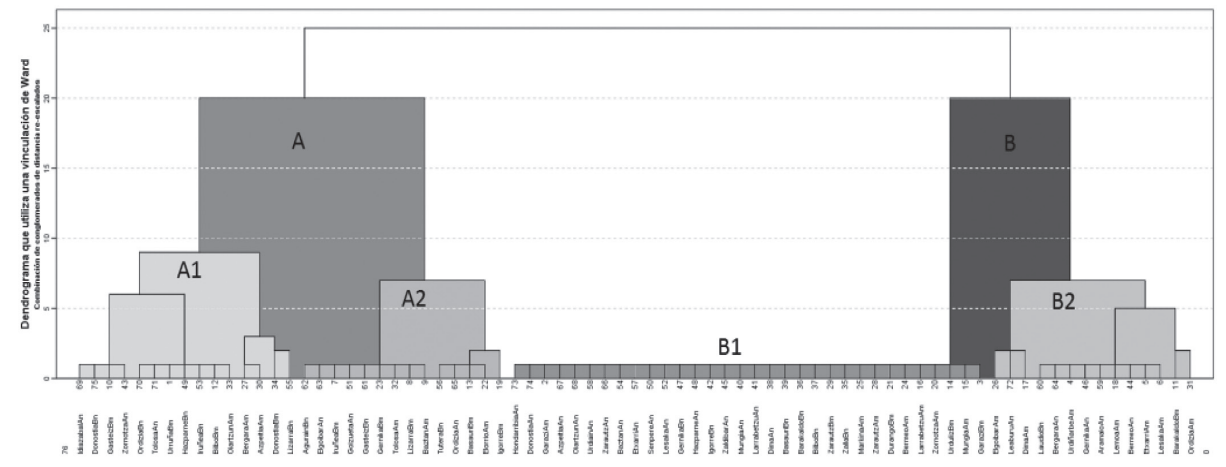

Figura 11. Dendograma en el que se reflejan los principales grupos resultantes del análisis de conglomerados jerárquico.

En el conglomerado A se sitúan 30 informantes (40\%) y en el conglomerado B los 45 restantes (60\%); a su vez, ambos conglomerados pueden dividirse en otros dos: A1 (16 informantes; 21,33 \%), A2 (14 informantes; 18,67\%), B1 (31 informantes; $41,33 \%)$ y B2 (14 informantes; $18,67 \%)$. En la matriz de datos se han introducido, como variables dicotómicas, las mismas variables que se han utilizado en la confección de los vectores.

En la Tabla II se muestra la distribución de las adscripciones de los informantes a cada conglomerado según el género de los mismos. Las diferencias que aparecen en la tabla no son estadísticamente significativas según la prueba de $\chi^{2}$.

Tabla II. Número y porcentaje de informantes adscritos a cada conglomerado según el género de los mismos.

\begin{tabular}{|l|r|r|r|r|}
\hline & \multicolumn{2}{|c|}{ Hombres } & \multicolumn{2}{c|}{ Mujeres } \\
\hline \multicolumn{1}{|c|}{ Grupo } & N & \multicolumn{1}{c|}{$\%$} & N & \multicolumn{1}{c|}{$\%$} \\
\hline A & 16 & 21,33 & 14 & 18,67 \\
\hline B & 18 & 24 & 27 & 36 \\
\hline
\end{tabular}

Si se observan las adscripciones de los informantes a los diferentes conglomerados, se aprecia que la mayoría de los que han aprendido el euskera en el entorno familiar se sitúan en el grupo B. La diferencia que se muestra en la Tabla III es 
estadísticamente significativa $\left(\chi^{2}=(\mathrm{gl}: 1) 9,595 ; \mathrm{p}=0,002\right)$.

Tabla III. Número y porcentaje de informantes adscritos a cada conglomerado según la lengua materna de los mismos.

\begin{tabular}{|c|c|c|c|c|}
\hline \multirow[b]{2}{*}{ Grupo } & \multicolumn{2}{|c|}{ Euskera } & \multicolumn{2}{|c|}{ Español } \\
\hline & $\mathrm{N}$ & $\%$ & $\mathrm{~N}$ & $\%$ \\
\hline A & 12 & 16 & 18 & 24 \\
\hline B & 34 & 45,33 & 11 & 14,67 \\
\hline
\end{tabular}

Finalmente, dividiendo el área donde se ha realizado la investigación en tres zonas principales: (1) occidental, comprendiendo las provincias de Araba y Bizkaia; (2) central, comprendiendo Gipuzkoa y Navarra; y (3) oriental, comprendiendo la zona del País Vasco continental se obtienen los resultados especificados en la Tabla IV. Como puede verse en ésta, mientras las diferencias en las distribuciones de los resultados por conglomerados en las zonas central y oriental son muy pequeñas, no sucede lo mismo con los resultados de la zona occidental, donde la mayoría de los informantes se incluyen en el conglomerado B.

Tabla IV. Número y porcentaje de informantes adscritos a cada conglomerado según la zona geográfica de procedencia de los mismos.

\begin{tabular}{|l|r|r|r|r|r|r|}
\hline & \multicolumn{2}{|c|}{$(1)$} & \multicolumn{2}{c|}{$(2)$} & \multicolumn{2}{|c|}{ (3) } \\
\hline & $\mathrm{N}$ & \multicolumn{1}{|c|}{$\%$} & $\mathrm{~N}$ & \multicolumn{1}{c|}{$\%$} & $\mathrm{~N}$ & \multicolumn{1}{c|}{$\%$} \\
\hline $\mathrm{A}$ & 9 & 27,27 & 18 & 51,43 & 3 & 42,86 \\
\hline B & 24 & 72,73 & 17 & 48,57 & 4 & 57,14 \\
\hline Total & 33 & 100 & 35 & 100 & 7 & 100 \\
\hline
\end{tabular}

\section{CONCLUSIONES}

Teniendo en cuenta los tres niveles lingüísticos, que son el léxico, sintáctico y el correspondiente al grupo prosódico no existe una gran variación con respecto a las cumbres tonales en cada sílaba tal y como se observa en la Figura 1 del apartado anterior; pero tratándose de un sistema complejo y adaptativo, con la interacción de los distintos niveles se muestra la complejidad de las realizaciones encontradas. En este contexto, las frecuencias de los quince vectores especificados como resultado del análisis de las interacciones entre los tres niveles son cambiantes, que es 
lo que lleva a percibir una melodía concreta en un texto o grupo prosódico. Siguiendo la idea de la no linealidad y transformación, se deben tener en cuenta las pequeñas diferencias que al combinarse pueden cambiar todo el sistema:

En los sistemas complejos, las pequeñas diferencias cuantitativas en ciertos parámetros a menudo provocan cambios de estado; es decir, diferencias cualitativas. Elman (2005) señala que las múltiples y pequeñas diferencias fenotípicas entre los seres humanos y otros primates (tales como el grado de sociabilidad, la atención compartida, la capacidad memorística, la habilidad para la secuenciación rápida, el control del tracto vocal, etc.) pueden tener, al combinarse, profundas consecuencias, resultando en formas de comunicación totalmente diferentes. A su vez, en un sistema dinámico, incluso cuando no se produzcan cambios paramétricos, en un cierto punto de la dinámica continua, el comportamiento del sistema puede cambiar drásticamente, llegando a una transformación o cambio de estado (Beckner, Blythe, Bybee, Christiansen, Croft, Ellis, Holland, Ke, Larsen-Freeman, Schoenemann, 2011: 14).

Respecto a las variables estudiadas, las diferencias no son significativas con respecto al género de los informantes en el empleo de la cumbre tonal en la lectura en voz alta, en cambio, la manera en la que se ha aprendido la lengua vasca (grupo A o B) sí influye en los resultados de las posiciones de las cumbres tonales, ya que la mayoría de los informantes que han participado en el estudio se agrupan en el conglomerado $\mathrm{B}$ al igual que aquellos informantes que proceden de la zona occidental del País Vasco. Estos resultados llevan a pensar que habría que medir por medio de test de percepción diseñados para el caso la sensación que produce este tipo de lecturas en voz alta en el oyente, para posteriormente poder crear y enseñar pautas específicas de lectura en voz alta.

Por último, sería deseable usar más enunciados del mismo tipo con otros elementos léxicos para poder observar y describir si el comportamiento descrito para el enunciado específico se repite en otros enunciados similares.

\section{REFERENCIAS}

Beckman, Mary y Pierrehumbert, Janet. (1986). Intonational Structure in Japanese and English. Phonology Yearbook. III. 15-70.

Beckner, Clay.; Blythe, Richard.; Bybee, Joan.; Christiansen, Morten H.; Croft, William.; Ellis, Nick C.; Holland, John.; Ke, Jinyun.; Larsen-Freeman, Diane, y Schoenemann, Tom. (2011). La lengua es un sistema adaptativo complejo. Lingüistica en la red. 1-25. Disponible en www.linred.es/articulos_pdf/LR_ articulo_04092011.pdf 
Benjamin, Rebekah George y Schwanenflugel, Paula J. (2010). Text complexity and oral reading prosody in young readers. Reading Research Quarterly, 45, 388-404.

Benjamin, Rebekah George; Schwanenflugel, Paula J.; Meisinger, Elizabeth B.; Groff, Carolyn.; Kuhn, Melanie R., y Steiner, Lilly. (2013). A spectrographically Grounded Scale for Evaluating Reading Expressiveness. Reading Research Quarterly, 48 (2), 105-133.

Boersma, Paul y Weenink, David. (2009). Praat: doing phonetics by computer, [Software package]. Disponible en http://www.fon.hum.uva.nl/praat/

Chomsky, Carol. (1978). When you still can't read in third grade: After decoding, what? In S.J. Samuels (ed.), What research has to say about reading instruction. (13-30) Newark, DE: International Reading Association.

Dowhower, Sarah L. (1991). Speaking of prosody: Fluency's unattended bedfellow. Theory Into Practice, 30, 165-175.

Elman, Jeffrey L. (2005). Connectionist views of cognitive development: Where next? Trends in Cognitive Science, 9, 111-117.

Elordieta, Gorka. (1997). Accent, tone and intonation in Lekeitio Basque. In F. Martínez-Gil, \& A. Morales-Front (eds.), Issues in the Phonology and Morphology of the Major Iberian Languages. Wahington, D.C.: Georgetown University Press.

Elordieta, Gorka. (1999). Primer estudio comparativo de la entonación de tres variedades dialectales vascas. In Actas del I Congreso de Fonética Experimental. (209-215). Barcelona: Universitat Rovira i Vigili y Universitat de Barcelona.

Elordieta, Gorka. (2007a). Constraints on intonational prominence of focalized constituents. In D. Büring., M. Gordon y C.L. Lee (eds.), Topic and focus: Papers from a workshop on intonation and meaning. Dordrecht: Springer.

Elordieta, Gorka. (2007b). A constraint-based analysis of the intonational realization of focus in Northerm Bizkaian Basque. In T. Riad., \& C. Gussenhoven (eds.), Tones and Tunes: Volume I, Typological Studies in Word and Sentence Prosody. Berlin: Mouton de Gruyter.

Fujisaki, Hiroya. (2004). Information, Prosody, and Modeling. Proceedings of Speech Prosody. Japón: Nara.

Gaminde, Iñaki. (1995). Bizkaieraren Azentu-Moldeez. Bilbao: Labayru Ikastegia. Gaminde, Iñaki. (1998). Euskaldunen azentuak. Bilbao: Labayru.

Gaminde, Iñaki. (2000). Azentu eta Intonazioa: egoera eta ikerketa baliabideak. In K. Zuazo (arg.), Dialektologia Gaiak. Araba: Arabako Foru Aldundia.

Gaminde, Iñaki. (2003). Intonazio ereduak Zeanurin. FLV, 93.

Gaminde, Iñaki. (2004a). Bilbotarron Euskararen Zuzuan CDa. Bilbao: Asapala Kultur Elkartea.

Gaminde, Iñaki. (2004b). Tonuak eta etenak Gatikako intonazioan. FLV, 97, 519-536. 
Gaminde, Iñaki. (2006). Intonazio kurben etenez. In J. Lakarra, \& J. I. Hualde (eds.), Studies in Basque and Historica Linguistics in Memory of R. L. Transk. R. L. Trasken oroitzapenetan ikerketak euskalaritza eta hizkuntzalaritza historikoaz. ASJU XL, 1-2, 351-376.

Gaminde, Iñaki. (2007). Tonuak eta etenak Bilboko gaztelaniaz eta euskaraz. Fontes linguae vasconum: Studia et documenta, 106, 455-474.

Gaminde, Iñaki. (2010). Bizkaiko Gazteen Prosodiaz: Euskaraz eta Gaztelaniaz. Bilbao: Mendebalde Kultura Alkartea y Bizkaiko Foru Aldundia.

Gaminde, Iñaki y Goikoetxea, Udane. (2005). Irakurketa Ozena ebaluatzeko irizpideak Bizkaian. Bilbao: Mendebalde Kultura Alkartea.

Gaminde, Iñaki; Etxebarria, Aintzane; Garay, Urtza, y Romero, Asier. (2012). Komunikazio gaitasunetik kultura arteko komunikazio gaitasunera: Baliabide didaktikoak. In Hizkuntzaz Jabetzen (209-251). Bilbao: Mendebalde Kultura Alkartea.

Gaminde, Iñaki; Etxebarria, Aintzane; Garay, Urtza, y Romero, Asier. (2013). La competencia prosódica en la lectura en voz alta: análisis de los aspectos rítmicos. Onomázein. 27 (1). 1-14.

Gaminde, Iñaki; Aurrekoetxea, Gotzon; Etxebarria, Aintzane; Garay, Urtza, y Romero, Asier. (2014). Ahoskera lantzeko argibideak eta jarduerak. Laguntzarako materiala: teoria eta praktika. Leioa: UPV/EHU.

Gaminde, Iñaki; Etxebarria, Aintzane; Romero, Asier, y Eguskiza, Naia. (2016). Diferencias en los tonos de frontera de los textos leídos en vasco por jóvenes bilingües. IV Congreso Internacional de Lingüistica y Literatura. Santander: Universidad de Cantabria (En prensa).

Gandarias, Rosa. (1996). Ispasterko Intonazioaren Deskribapena: Azterketa Fonetikoa. Tesis Doctoral. País Vasco: UPV/ EHU.

Gil, Juana. (2007). Fonética para profesores de español: De la teoría a la práctica. Madrid: Arco/Libros.

Hualde, José Ignacio. (1999). From phrase-final to post-initial accent in western basque. (Sin publicar).

Hualde, José Ignacio. (2003). El modelo métrico y autosegmental. In P. Prieto (ed.), Teorías de la Entonación. (155-184). Barcelona: Ariel Lingüistica.

Hualde, José Ignacio; Elordieta, Gorka; y Elordieta, Arantzazu. (1994). The Basque Dialect of Lekeitio. Bilbao y Donostia: Servicio Editorial de la Universidad del País Vasco y Diputación Foral de Gipuzkoa.

Ito, Kiwako. (2002). The effect of focus on lexical pitch peak alignment in Tokyo Japanese and Bermeo Basque. Tokio, LP2002.

Ito, Kiwako. (2003). The focus-independent effect of tonal proximity on the realization of lexical pitch accent in Tokyo Japanese and Bermeo Basque. In M.J. Solé, D. Recasens, y J. Romero (eds.), Proceedings of the 15th International Congress of Phonetic Sciences. Barcelona. 
Jun, S.A. y Elordieta, Gorka. (1997). Intonational Structure of Lekeitio Basque. In A. Botinis, G. Kouroupetroglou, \& G. Carayiannis (eds.), Intonation: Theory, Models and Applications, Proceedings of an ESCA Workshop (193-196). Atenas: ESCA.

Klauda, Susan L. y Guthrie, John T. (2008). Relationships of three components of reading fluency to reading comprehension. Journal of Educational Psychology, 100, 310-321.

Kuhn, Melanie R. y Stahl, Steven. A. (2003). A review of developmental and remedial practices. Journal of Educational Psychology 95, 3-21.

Liberman, Mark y Sag, I. (1975). Intonational Disambiguation of Indirect Speech Acts. CLS, 11, 487-497.

Llisterri, Joaquim. (2016). Los elementos suprasegmentales. Disponible en http:// liceu.uab.es/_joaquim/phonetics/fon_prosod/suprasegmentales.html

Magro Eizmendi, Imanol. (2013). Bildumako maisulana. Noticia publicada en Berria.eus el 15/12/2013. Recuperada de http://www.berria.eus/paperekoa/1822/021/001/2013-12-15/bildumako_maisulana.htm.

Miller, Justin y Schwanenflugel, Paula J. (2006). Prosody of syntactically complex sentences in the oral reading of young children. Journal of Educational Psychology, 98 (4), 839-853.

Miller, Justin y Schwanenflugel, Paula J. (2008). A longitudinal study of the development of reading prosody as a dimension of oral reading fluency in early elementary school children. Reading Research Quarterly, 43 (4), 336-354.

Mozziconacci, Sylvie. (1998). Speech Variability and Emotion: Production and Perception. Eindhoven: Proefschrift.

Navas, Eva. (2003). Modelado Prosódico del Euskera Batúa para Conversión de Texto a Habla. Tesis Doctoral. Bilbao: Universidad del país Vasco/Euskal Herriko Unibertsitatea (UPV/EHU).

Nespor, Marina y Vogel, Irene. (2007). Prosodic phonology with a new foreword. Studies in generative grammar. Berlin: Mouton de Gruyter.

Pierrehumbert, Janet. (1980). The phonology and phonetics of English intonation. Tesis doctoral. Indiana University Linguistics Club: MIT Press.

Pierrehumbert, Janet y Beckman, Mary. (1988). Japanese tone structure. Cambridge: MIT Press.

Prieto, Pilar. (2002). Entonació. Models, teoria, mètodes. Barcelona: Ariel Lingüística.

Rasinski, Timothy V. (1990). Investigating measures of reading fluency. Educational Research Quarterly, 14 (3), 37-44.

Rasinski, Timothy V. (2004). Assessing Reading Fluency. Honolulu: Pacific Resources for Education and Learning.

Rasinski, Timothy V.; Rikli, Andrew, y Johnston, Susan. (2009). Reading fluency: More than automaticity? More than a concern for the primary grades? Literacy 
Research and Instruction, 48, 350-361.

Samuels, SJ.; Schermer, N., y Reinking, D. (1992). Reading fluency: Techniques for making decoding automatic. In S.J. Samuels, A.E. Farstrup (eds.), What research has to say about reading instruction. (124-144). Newark, DE: International Reading Association.

Schwanenflugel, Paula J.; Hamilton, Anne M.; Wisenbaker, Joseph M.; Kuhn, Melanie R., y Stahl, Steven A. (2004). Becoming a fluent reader: Reading skill and prosodic features in the oral reading of young readers. Journal of Educational Psychology, 96 (1), 119-129.

Uriarte, Jon Andoni. (1995). Fruizko Hizkera: Azterketa Linguistikoa. Tesis Doctoral. Bilbao: Universidad de Deusto.

Uriarte, Jon Andoni. (2004). Fruiztarrak Berbetan. Bilbao: Fruizko Udala.

Veenendaal, Nathalie J.; Groen, Margriet A., y Verhoeven, L. (2014). The role of speech prosody and text Reading prosody in children's reading comprehension. British Journal of Educational Psychology, 84, 521-536. 